Este documento se trata del preprint de la comunicación titulada "La eficiencia de las bibliotecas públicas y universitarias de Cataluña: ¿Modelos de buenas prácticas? Evaluación y análisis a partir de la herramienta Secaba-Rank"

Autores: Pedro Lázaro-Rodríguez (pedrolr@ugr.es), Javier López-Gijón, Enrique Herrera-Viedma

Dicha comunicación se presentará en el IX Encuentro Ibérico de la Asociación de Educación e Investigación en Ciencia de la Información de Iberoamérica y el Caribe (EDICIC) tendrá lugar en la Universitat de Barcelona, del 9 al 11de Julio de 2019, bajo el lema "Datos abiertos e inclusión digital en la era del Big Data". 


\title{
La eficiencia de las bibliotecas públicas y universitarias de Cataluña: ¿Modelos de buenas prácticas? Evaluación y análisis a partir de la herramienta Secaba-Rank
}

\author{
Pedro Lázaro Rodríguez ${ }^{1}$, Javier López Gijón², Enrique Herrera Viedma ${ }^{3}$ \\ ${ }^{1}$ Código ORCID: http://orcid.org/0000-0002-8756-0507. Universidad de Granada. Departamento \\ de Información y Comunicación. Campus Universitario de Cartuja. Edificio Máximo. 18071 \\ Granada, España. Correo electrónico: pedrolr@ugr.es. \\ ${ }^{2}$ Código ORCID: http://orcid.org/0000-0001-6517-8991. Universidad de Granada. Departamento \\ de Información y Comunicación. Campus Universitario de Cartuja. Edificio Máximo. 18071 \\ Granada, España. Correo electrónico: jgijon@ugr.es. \\ 3 Código ORCID: Universidad de Granada, Departamento de Ciencias de la Computación e \\ Inteligencia Artificial. Periodista Daniel Saucedo Aranda, s/n. 18071 Granada, España. Correo \\ electrónico: viedma@decsai.ugr.es.
}

Tipo de contribución: comunicación

Palabras clave: Bibliotecas públicas; Bibliotecas universitarias; Cataluña; Secaba-Rank; Evaluación de bibliotecas; Eficiencia; Indicadores de segundo nivel.

\section{Contexto del trabajo}

A finales de 2016 se desarrolló e implementó Secaba-Rank ${ }^{1}$, herramienta abierta y en línea para la evaluación de las redes de lectura pública (bibliotecas públicas) y las bibliotecas universitarias de España. La característica más original de dicha herramienta fue el desarrollo de indicadores de segundo nivel para medir la eficiencia de bibliotecas gracias a la metodología basada en la teoría general de sistemas. Con todo, la herramienta supuso la elaboración de un ranking de eficiencia con actualización anual que permite la identificación de los modelos de buenas y el benchmarking en aras a la mejora especialmente de los sistemas o bibliotecas que más lo necesitan. La herramienta fue presentada y difundida a la comunidad científica a inicios del año 2018 (Lázaro-Rodríguez, López-Gijón, Alonso, Martínez-Sánchez y Herrera-Viedma, 2018).

El actual trabajo que se presenta en forma de comunicación lleva por título "La eficiencia de las bibliotecas públicas y universitarias de Cataluña: ¿Modelos de buenas prácticas? Un análisis y evaluación a partir de la herramienta Secaba-Rank". En dicha comunicación se presentan los resultados de Secaba-Rank para los años más recientes (2016 en bibliotecas públicas y 2017 en las universitarias), atendiendo concretamente a los resultados de las bibliotecas de Cataluña. No se trata únicamente de la mera presentación de resultados, sino de un análisis de los mismos poniéndolos en relación con los de otras redes de lectura pública y bibliotecas universitarias. Así, se da respuesta a la cuestión incluida en el título sobre si las bibliotecas de Cataluña son modelos de buenas prácticas o no. En ese sentido, se exponen también las relaciones de las bibliotecas catalanas con las demás y se ofrece información de cómo podrían mejorar y como podrían servir de modelo de buenas prácticas a otras para su mejora.

1Acceso desde: http://secaba.ugr.es/rank/ 


\section{Objetivos y preguntas de investigación}

Los objetivos del trabajo pueden entenderse como la respuesta a las siguientes preguntas de investigación:

- ¿Son eficiente las redes de lectura pública y las bibliotecas universitarias de Cataluña?

- ¿Son modelos de buenas prácticas para el resto de bibliotecas de España?

- ¿Cómo podrían mejorar las bibliotecas catalanas y como podrían servir de modelo para la mejora de otras?

La respuesta a cada pregunta de investigación se lleva a cabo en un ejercicio de discusión reflexionando y aportando razones sólidas para las conclusiones que se alcanzan. El trabajo consta, además del contexto y los objetivos, de una sección para los materiales y metodología, otra para los resultados, y una última para la discusión y conclusiones.

\section{Materiales y metodología}

Los materiales utilizados son los resultados de la herramienta Secaba-Rank para las bibliotecas públicas españolas (Secaba-Rank Públicas ${ }^{2}$ ) y las universitarias (Secaba-Rank Universitarias ${ }^{3}$ ). Para las primeras, se consideran resultados a nivel de comunidades autónomas y provincias para el año 2016, y para las universitarias, del año 2017 (últimos datos disponibles para cada caso).

La metodología de la herramienta fue expuesta y puede ser consultada en Lázaro-Rodríguez et al. (2018), además de estar disponible en la sección de la metodología de la herramienta ${ }^{4}$. A su vez, dicha metodología fue sometida a examen en otro trabajo científico con el objetivo de probar su validez (Lázaro-Rodríguez et al., 2019). Para ello, se llevó a cabo una comparación de los resultados de eficiencia según la metodología Secaba-Rank con los de la técnica DEA de análisis envolvente de datos y el calificado como método Finlandia, concluyendo que los 3 métodos miden la eficiencia de las bibliotecas. En esta sección se incluyen contenidos de ambos trabajos de investigación para explicar la metodología.

En este caso concreto y debido al análisis que se pretende llevar a cabo, se da especial importancia a los indicadores de la potencia y la formulación del sistema, pues son los que ofrecen información sintética y precisa acerca del benchmarking y la identificación y comparación de las bibliotecas que pueden considerarse los modelos de buenas prácticas.

El indicador de la potencia del sistema pone en relación las entradas (presupuesto) de los sistemas biblioteca con las salidas (préstamos), y resulta de la división de la potencia de salida entre la de entrada. $\mathrm{Su}$ fórmula es la siguiente:

$P($ sis $)=\frac{P(\text { sal })}{P(\text { ent })}$

Donde:

2 Acceso desde: http://secaba.ugr.es/rank/publicas/

3 Acceso desde: http://secaba.ugr.es/rank/universitarias/

4 Acceso desde: http://secaba.ugr.es/rank/index.php/metodologia 
$P($ sis $)=$ Potencia del sistema

$P($ sal $)=$ Potencia de salida

$P($ ent $)=$ Potencia de entrada

Además, permite clasificar los sistemas según 3 estados posibles: potencia (cuando el sistema produce mayor nivel de salidas que de entradas, y por ello, eficiente), dispotencia (cuando el sistema produce menor nivel de salidas que de entradas, y por ello, no eficiente), y sin potencia (cuando el sistema produce el mismo nivel de salidas que de entradas). Los estados pueden verse en la Tabla 1:

Tabla 1. Estados posibles de una biblioteca según la potencia del sistema

\begin{tabular}{|c|c|c|c|}
\hline $\begin{array}{l}\text { Potencia del } \\
\text { sistema }\end{array}$ & Descripción del sistema & Formulación & Estado \\
\hline$P($ sis $)>1$ & $\begin{array}{l}\text { Se producen más salidas que } \\
\text { entradas se le suministran }\end{array}$ & $P($ sal $)>P($ ent $)$ & Potencia \\
\hline$P($ sis $)=1$ & $\begin{array}{l}\text { Se producen las mismas salidas } \\
\text { que entradas }\end{array}$ & $P($ sal $)=P($ ent $)$ & Sin potencia \\
\hline$P($ sis $)<1$ & $\begin{array}{l}\text { Se producen menos salidas que } \\
\text { entradas se le suministran }\end{array}$ & $P($ sal $)<P($ ent $)$ & Dispotencia \\
\hline
\end{tabular}

El indicador de la formulación del sistema consiste, valga la redundancia, en una formulación del indicador de la potencia del sistema. Se escribe en primer lugar la potencia de entrada, junto con una flecha hacia la potencia de salida, que aparece en último lugar (Potencia de entrada $\rightarrow$ Potencia de salida).

$F($ sis $)=P($ ent $) \rightarrow P($ sal $)$

Donde:

$F($ sis $)=$ Formulación del sistema

$P($ ent $)=$ Potencia de entrada

$P($ sal $)=$ Potencia de salida

La formulación del sistema permite clasificar los sistemas por potencia de entrada o de salida e identificar claramente los que, teniendo un mismo nivel de entradas (o salidas), producen mayores o menores salidas (o entradas). Es decir, gracias a este indicador se pueden analizar los sistemas que tienen similares entradas (presupuesto) para ver cómo las han procesado en el nivel de salidas (préstamos). Así, los sistemas que, teniendo un nivel de entradas similar, consiguen mayores salidas (préstamos) que otros, se convierten así en los modelos de buenas prácticas para los que consiguen menor nivel de salidas.

Con todo, el análisis que se plantea permite dar respuesta a las preguntas de investigación expuestas en la sección sobre los objetivos.

\section{Resultados}


En aras a una mayor claridad en la exposición de los resultados, se han seleccionado partes concretas de las tablas incluidas en Secaba-Rank, pues incluir tablas de los resultados especialmente con todas las provincias y bibliotecas universitarias, dificultaría la apreciación de los mismos. Los resultados seleccionados para las redes de lectura pública por comunidades autónomas del año 2016 en Secaba-Rank Públicas pueden verse en la Tabla 2.

Tabla 2: Eficiencia y resultados para las redes de lectura pública por comunidades autónomas (CCAA) 2016

\begin{tabular}{lcccccc}
\hline \multicolumn{1}{c}{ Red } & Pres pc & Circ pc & Form(sis) & P(sis) & Ranking & Estado \\
\hline C. F. de Navarra & 6.57 & 1.35 & $0.83 \rightarrow 1.27$ & 1.54 & $1 / 17$ & Potencia \\
Castilla y León & 9.87 & 1.86 & $1.24 \rightarrow 1.75$ & 1.41 & $2 / 17$ & Potencia \\
La Rioja & 7.42 & 1.37 & $0.93 \rightarrow 1.29$ & 1.38 & $3 / 17$ & Potencia \\
P. de Asturias & 6.51 & 1.16 & $0.82 \rightarrow 1.09$ & 1.33 & $4 / 17$ & Potencia \\
Cataluña & $\mathbf{1 1 . 2 1}$ & $\mathbf{1 . 9 4}$ & $\mathbf{1 . 4 1 \rightarrow 1 . 8 3}$ & $\mathbf{1 . 3 0}$ & $\mathbf{5 / 1 7}$ & Potencia \\
Total Redes & 7.95 & 1.06 & $1.00 \rightarrow 1.00$ & 1.00 & Base & Base \\
Castilla-La Mancha & 11.06 & 1.35 & $1.39 \rightarrow 1.27$ & 0.91 & $11 / 17$ & Dispotencia \\
País Vasco & 13.82 & 1.41 & $1.74 \rightarrow 1.33$ & 0.76 & $15 / 17$ & Dispotencia \\
\hline
\end{tabular}

Nota: Pres pc equivale a presupuesto per cápita; Circ pc, a circulación (préstamos) per cápita; Form(sis) a la formulación del sistema y P(sis) a la potencia del sistema. El 17 de la columna Ranking se refiere al total de comunidades autónomas que incluye Secaba-Rank Públicas.

Como puede verse, el sistema o red Cataluña tiene una Potencia del sistema de 1.30, resultando quinta en el ranking por comunidades autónomas de un total de 17. Dado que su resultado es mayor que 1, su estado es de potencia. Es decir, su nivel de salidas es mayor que el de entradas, y por eso puede considerarse un sistema eficiente en relación al resto de comunidades autónomas.

Atendiendo a su Formulación del sistema, se aprecia que su Potencia de entrada es de 1.41 y la de salida de 1.83. En ese sentido y para la identificación de los modelos de buenas prácticas, se puede traer a escena al sistema Castilla La-Mancha. Este sistema tiene una Potencia de entrada de 1.39, similar a la de Cataluña. Sin embargo, mientras que Cataluña, con ese nivel de entradas obtiene 1.83 de salidas, Castilla La-Mancha obtiene 1.27 de salidas. Por tanto, Cataluña es un modelo de buenas prácticas para Castilla La-Mancha. Este hecho también puede verse atendiendo a los resultados del presupuesto y la circulación per cápita de ambos sistemas: el presupuesto de ambos es similar (11.21 para Cataluña y 11.06 para Castilla La-Mancha), pero invirtiendo lo mismo, Cataluña alcanza 1.94 préstamos per cápita mientras que Castilla La-Mancha 1.35. De ahí que Cataluña sea modelo de buenas prácticas para Castilla La-Mancha.

A su vez, si se focaliza la atención en la Potencia de salida de la Formulación del sistema, se observa que la de Cataluña es de 1.83. Un sistema con un resultado similar es el de Castilla y León, con una Potencia de salida de 1.75, algo menos que Cataluña pero cercana. Pero sucede que mientras que Cataluña lo consigue con 1.41 en el nivel de entradas (Potencia de entrada), Castilla y León lo consigue con 1.24. Por ello Castilla y León sería un sistema más eficiente que Cataluña, convirtiéndose en su modelo de buenas prácticas. Por tanto, a nivel de comunidades autónomas, Cataluña es modelo de buenas prácticas y a la vez puede atender a otros sistemas modelo para sí 
misma.

El mismo análisis anterior puede hacerse a nivel de provincias. La selección de resultados para las redes de lectura pública por provincias del año 2016 en Secaba-Rank Públicas puede verse en la Tabla 3.

Tabla 3: Principales resultados para las redes de lectura pública por provincias 2016

\begin{tabular}{lcccccc}
\hline \multicolumn{1}{c}{ Red } & Pres pc & Circ pc & Form(sis $)$ & P(sis $)$ & Ranking & Estado \\
\hline Soria & 13.41 & 4.96 & $1.69 \rightarrow 4.67$ & 2.77 & $1 / 50$ & Potencia \\
Segovia & 8.08 & 1.87 & $1.02 \rightarrow 1.76$ & 1.73 & $2 / 50$ & Potencia \\
Lleida & $\mathbf{8 . 1 3}$ & $\mathbf{1 . 5 8}$ & $\mathbf{1 . 0 2} \rightarrow \mathbf{1 . 4 8}$ & $\mathbf{1 . 4 5}$ & $\mathbf{6} / \mathbf{5 0}$ & Potencia \\
La Rioja & 7.42 & 1.37 & $0.93 \rightarrow 1.29$ & 1.38 & $8 / 50$ & Potencia \\
Tarragona & $\mathbf{7 . 8 8}$ & $\mathbf{1 . 4 3}$ & $\mathbf{0 . 9 9} \rightarrow \mathbf{1 . 3 5}$ & $\mathbf{1 . 3 6}$ & $\mathbf{9 / 5 0}$ & Potencia \\
Barcelona & $\mathbf{1 1 . 7 3}$ & $\mathbf{2 . 0 5}$ & $\mathbf{1 . 4 8} \rightarrow \mathbf{1 . 9 2}$ & $\mathbf{1 . 3 0}$ & $\mathbf{1 2 / 5 0}$ & Potencia \\
Huesca & 11.52 & 1.85 & $1.45 \rightarrow 1.74$ & 1.20 & $14 / 50$ & Potencia \\
Zamora & 13.10 & 2.05 & $1.65 \rightarrow 1.93$ & 1.17 & $15 / 50$ & Potencia \\
Girona & $\mathbf{1 2 . 6 2}$ & $\mathbf{1 . 9 4}$ & $\mathbf{1 . 5 9} \rightarrow \mathbf{1 . 8 2}$ & $\mathbf{1 . 1 5}$ & $\mathbf{1 6 / 5 0}$ & Potencia \\
Total Redes & 7.95 & 1.06 & $1.00 \rightarrow 1.00$ & 1.00 & Base & Base \\
Palencia & 12.39 & 1.60 & $1.56 \rightarrow 1.50$ & 0.96 & $22 / 50$ & Dispotencia \\
Cáceres & 7.52 & 0.90 & $0.95 \rightarrow 0.84$ & 0.89 & $26 / 50$ & Dispotencia \\
Ávila & 12.05 & 1.42 & $1.52 \rightarrow 1.34$ & 0.88 & $27 / 50$ & Dispotencia \\
Badajoz & 8.14 & 0.88 & $1.02 \rightarrow 0.83$ & 0.81 & $29 / 50$ & Dispotencia \\
\hline
\end{tabular}

Tal y como se observa, todas las provincias de Cataluña son sistemas potentes y por ello eficientes. Siguiendo el análisis llevado a cabo a partir de la Tabla 2 a nivel de comunidades autónomas y nombrando algunos ejemplos, Lleida sería modelo de buenas prácticas para Badajoz, Tarragona para Cáceres, Barcelona para Ávila y Huesca, y Girona para Palencia. Por otro lado, algunos modelos de buenas prácticas para las provincias de Cataluña serían Segovia para Tarragona y Lleida, Lleida para Tarragona, o Soria y Zamora para Girona.

En tercer lugar, el análisis puede plantearse también en las bibliotecas universitarias de Cataluña incluidas en Secaba-Rank. La selección de resultados para bibliotecas universitarias del año 2017 en Secaba-Rank Universitarias puede verse en la Tabla 4.

Tabla 4: Principales resultados para las bibliotecas universitarias 2017

\begin{tabular}{lcccccc}
\hline \multicolumn{1}{c}{ Biblioteca } & Pres pc & Circ pc & Form(sis) & P(sis) & Ranking & Estado \\
\hline U. Autònoma de Barcelona & $\mathbf{2 2 7 . 0 3}$ & $\mathbf{6 9 . 9 0}$ & $\mathbf{1 . 1 9} \rightarrow \mathbf{2 . 2 3}$ & $\mathbf{1 . 8 7}$ & $\mathbf{1 / 5 8}$ & Potencia \\
U. Politècnica de València & 167.01 & 46.79 & $0.88 \rightarrow 1.49$ & 1.70 & $3 / 58$ & Potencia \\
U. Pablo de Olavide & 160.44 & 42.73 & $0.84 \rightarrow 1.36$ & 1.62 & $4 / 58$ & Potencia
\end{tabular}




\begin{tabular}{lcccccc} 
U. Autónoma de Madrid & 229.47 & 56.36 & $1.20 \rightarrow 1.80$ & 1.49 & $5 / 58$ & Potencia \\
U. de Sevilla & 209.77 & 48.92 & $1.10 \rightarrow 1.56$ & 1.42 & $6 / 58$ & Potencia \\
U. Rovira i Virgili & $\mathbf{2 0 8 . 5 6}$ & $\mathbf{4 6 . 2 8}$ & $\mathbf{1 . 0 9} \rightarrow \mathbf{1 . 4 7}$ & $\mathbf{1 . 3 5}$ & $\mathbf{8} / \mathbf{5 8}$ & Potencia \\
U. de Barcelona & $\mathbf{1 6 5 . 8 6}$ & $\mathbf{3 6 . 6 8}$ & $\mathbf{0 . 8 7} \rightarrow \mathbf{1 . 1 7}$ & $\mathbf{1 . 3 4}$ & $\mathbf{9 / 5 8}$ & Potencia \\
U. de Cádiz & 197.16 & 42.06 & $1.03 \rightarrow 1.34$ & 1.30 & $11 / 58$ & Potencia \\
U. Pompeu Fabra & $\mathbf{3 6 2 . 7 4}$ & $\mathbf{6 9 . 4 6}$ & $\mathbf{1 . 9 0} \rightarrow \mathbf{2 . 2 1}$ & $\mathbf{1 . 1 6}$ & $\mathbf{1 3 / 5 8}$ & Potencia \\
Total Rebiun & 190.56 & 31.39 & $1.00 \rightarrow 1.00$ & 1.00 & Base & Base \\
U. Politècnica de Catalunya & $\mathbf{1 9 8 . 7 9}$ & $\mathbf{3 1 . 3 9}$ & $\mathbf{1 . 0 4} \rightarrow \mathbf{1 . 0 0}$ & $\mathbf{0 . 9 6}$ & $\mathbf{1 7 / 5 8}$ & Dispotencia \\
U. de Navarra & 374.60 & 59.14 & $1.97 \rightarrow 1.88$ & 0.96 & $17 / 58$ & Dispotencia \\
U. de Jaén & 229.09 & 35.89 & $1.20 \rightarrow 1.14$ & 0.95 & $18 / 58$ & Dispotencia \\
U. Intern.de Catalunya & $\mathbf{1 2 1 . 1 8}$ & $\mathbf{1 8 . 0 4}$ & $\mathbf{0 . 6 4} \rightarrow \mathbf{0 . 5 7}$ & $\mathbf{0 . 9 0}$ & $\mathbf{2 1 / 5 8}$ & Dispotencia \\
U. de Alcalá & 160.12 & 21.71 & $0.84 \rightarrow 0.69$ & 0.82 & $24 / 58$ & Dispotencia \\
U. de Girona & $\mathbf{1 9 7 . 9 5}$ & $\mathbf{2 5 . 2 9}$ & $\mathbf{1 . 0 4 \rightarrow \mathbf { 0 . 8 1 }}$ & $\mathbf{0 . 7 8}$ & $\mathbf{2 6 / 5 8}$ & Dispotencia \\
U. Complutense & 203.61 & 25.32 & $1.07 \rightarrow 0.81$ & 0.75 & $28 / 58$ & Dispotencia \\
U. Castilla-La Mancha & 199.93 & 24.37 & $1.05 \rightarrow 0.78$ & 0.74 & $29 / 58$ & Dispotencia \\
U. de Lleida & $\mathbf{2 0 9 . 1 0}$ & $\mathbf{2 4 . 4 5}$ & $\mathbf{1 . 1 0} \rightarrow \mathbf{0 . 7 8}$ & $\mathbf{0 . 7 1}$ & $\mathbf{3 1 / 5 8}$ & Dispotencia \\
U. Politécnica de Cartagena & 168.32 & 19.05 & $0.88 \rightarrow 0.61$ & 0.69 & $32 / 58$ & Dispotencia \\
Mondragon U. & 124.18 & 11.05 & $0.65 \rightarrow 0.35$ & 0.54 & $38 / 58$ & Dispotencia \\
\hline
\end{tabular}

Como se puede ver, las bibliotecas de la U. Autònoma de Barcelona, U. Rovira i Virgili, U. de Barcelona y U. Pompeu Fabra son sistemas potentes y por ello considerados como eficientes. Cabe destacar que la de la U. Autònoma de Barcelona es la primera en el ranking total de Secaba-Rank y por ello la más eficiente de todas las incluidas en la herramienta. Por su lado, las bibliotecas de la U. de Lleida, U. de Girona, U. Internacional de Catalunya y U. Politècnica de Catalunya son sistemas dispotentes y por tanto no eficientes.

Siguiendo el análisis presentado al comentar la Tabla 2 y como ejemplo, las bibliotecas potentes universitarias de Cataluña serían modelo de buenas prácticas para algunas otras bibliotecas muy concretamente de la siguiente forma: la U. Autònoma de Barcelona lo sería para la de Sevilla y Jaén al tener un nivel de entradas similar per alcanzar un nivel de salidas más altos; de la misma forma, la de la U. Rovira i Virgili lo sería para la de Lleida y la Complutense; la de la U. de Barcelona, para las de la U. de Alcalá y la de la U. Politécnica de Cartagena; y la de la U. Pompeu Fabra, para la U. de Navarra (privada).

Pero también pueden identificarse modelos de buenas prácticas para algunas de las bibliotecas potentes de Cataluña. Así, la de la U. Pablo Olavide sería un modelo de buenas prácticas para la de la U. de Barcelona, pues con unas mismas entradas ( 0.84 y 0.87 en la Potencia de entrada respectivamente), la primera alcanza 1.36 en salidas mientras que la segunda 1.17 . No obstante ambas son potentes y por ello eficientes.

En cuanto a las bibliotecas universitarias de Cataluña dispotentes, sus modelos de buenas prácticas quedarían de la siguiente forma: para las de las U. de Girona y U. Politècnica de Catalunya, la de 
Cádiz sería un modelo al conseguir mayores salidas con similares entradas (Cádiz con 1.03 en entradas y las 2 de Cataluña con 1.04, y Cádiz con 1.34 en salidas y Girona con 0.81 y la Politècnica de Catalunya con 1, todo en potencia de entrada y salida respectivamente). A su vez y como se añadió más arriba, la de Rovira i Virgili sería un modelo de buenas prácticas para la de Lleida, pues con un nivel de entradas similar (1.09 y 1.1 respectivamente), obtiene un mayor nivel en la potencia de salida ( 1.47 y 0.71 respectivamente)

\section{Discusión y conclusiones}

Los objetivos de este trabajo se plantearon como respuesta a 3 preguntas de investigación. La primera de ellas consistía en si son eficiente las redes de lectura pública y las bibliotecas universitarias de Cataluña o no. Para ello, se atendió a los resultados de la potencia del sistema y al estado de los sistemas de Cataluña. La segunda pregunta planteaba si los sistemas catalanes son modelos de buenas prácticas para el resto de bibliotecas de España. Al respecto, se llevó a cabo un análisis centrado en el indicador de la formulación del sistema. En cuanto a la tercera pregunta de investigación, aludía a cómo podrían mejorar las bibliotecas catalanas y como podrían servir de modelo para la mejora de otras.

En relación a la primera pregunta de investigación, los resultados permiten establecer que a nivel de bibliotecas públicas tanto por comunidades autónomas como por provincias las bibliotecas de Cataluña son eficientes. Por su lado, las bibliotecas universitarias de Cataluña eficientes son las de la U. Autònoma de Barcelona, U. Rovira i Virgili, U. de Barcelona y U. Pompeu Fabra, mientras que son no eficientes las de la U. de Lleida, U. de Girona, U. Internacional de Catalunya y U. Politècnica de Catalunya.

En relación a la segunda pregunta de investigación, en la sección de resultados se mostró para qué bibliotecas podrían considerarse modelos de buenas prácticas las de Cataluña y cuáles lo podrían ser para ellas. Los resultados hallados permiten establecer que efectivamente pueden ser considerados modelos de buenas prácticas para otros sistemas, pero a su vez los hay que servirán de modelos a los catalanes.

Por último, en cuanto a la tercera pregunta de investigación, identificados los modelos de buenas prácticas, sería el momento de atender a las diferencias para encontrar formas de mejora entre los sistemas. Para ello, podrían llevarse a cabo evaluaciones cuantitativas analizando las diferencias, por ejemplo, entre el número de personal por habitantes, el contexto social del entorno en que se hallan las bibliotecas, el área disponible en las distintas bibliotecas, la colección per cápita, etc., para tratar de identificar los puntos débiles y también las fortalezas. En ese sentido, algunas investigaciones ya publicadas tratan sobre la modelización del uso de las bibliotecas (préstamos y visitas) para conocer los factores que serían los predictores para aumentar el uso (Lázaro-Rodríguez y Vakkari, 2018).

A su vez, podrían llevarse a cabo evaluaciones de naturaleza subjetiva, como por ejemplo, estudios de satisfacción de personas usuarias basados en la metodología Libqual para conocer el grado de satisfacción entre las usuarias de bibliotecas de distintas comunidades autónomas o provincias y bibliotecas universitarias. Estos tipos de estudio ofrecen información muy útil sobre lo que la persona usuaria cree necesario mejorar considerando a la biblioteca según las dimensiones del valor afectivo del personal, la biblioteca como espacio y el control de la información. Así, 
llevando a cabo estudios Libqual comparando bibliotecas de diferentes lugares, podrían analizarse las diferencias y considerarlas como punto del que partir hacia la mejora.

Con todo, los autores de la comunicación atenderemos también al debate que se genere en el IX Congreso EDICIC 2019 al presentar este trabajo tras ser expuesto a profesionales del área para compartir ideas sobre evaluación de bibliotecas y con la consecuencia de aumentar el conocimiento del tema en concreto y la disciplina de la Biblioteconomía y la Documentación en general.

\section{Referencias}

- Lázaro-Rodríguez, P., López-Gijón, J., Alonso, S., Martínez-Sánchez, M.-Á., y HerreraViedma, E. (2018). Secaba-Rank, herramienta online para analizar y evaluar bibliotecas. El profesional de la información, 27(2), 278-288. doi: https://doi.org/10.3145/epi.2018.mar.06

- Lázaro-Rodríguez, P., López-Gijón, J., y Herrera-Viedma, E. (2019). Secaba-Rank a examen: validación de su metodología sobre eficiencia en bibliotecas versus la técnica Data Envelopment Analysis (DEA) y el método Finlandia. El profesional de la información, v. 28, n. 3, e280316. doi: https://doi.org/10.3145/epi.2019.may.16

- Lázaro-Rodríguez, P., y Vakkari, P. (2018). Modelizando el uso de las bibliotecas públicas a través de sus características: estudio comparativo entre España y Finlandia para los préstamos y las visitas. Revista española de documentación científica, v. 41, n. 4, p. e216. doi: https://doi.org/10.3989/redc.2018.4.1544 